\title{
Local metothrexate treatment of cesarean scar ectopic pregnancy
}

\author{
Davut Güven, Kadir Bakay*, A. Sertaç Batığlu \\ Department of Obstetrics and Gynecology, Faculty of Medicine, Ondokuz Mayis University, Samsun, Turkey \\ Email: *drkadirbakay@gmail.com
}

Received 22 July 2012; revised 20 August 2012; accepted 30 August 2012

\begin{abstract}
Myometrial pregnancy developing in a previous caesarean section scar is the rarest of all ectopic pregnancies (EP) and probably one of the most dangerous of all because of the risk of rupture and hemorrhage. The recent recognition of this problem means that diagnosis and management are still in their infancy, and there is no consensus regarding the best management of CSP. Methotrexate (MTX) can be administered systemically or locally, or in both ways, with the aid of ultrasound. Patients diagnosed with caesarean scar pregnancies (CSP) in our clinic underwent transvaginal treatment of ectopic pregnancy. Safe and short treatment under transvaginal ultrasonographic (USG) guidance was performed uneventfully in all cases. The operating time ranged from 5 to 10 minutes with no blood loss. Serum $\beta$-hCG ( $\beta$-subunit of human chorionic gonadotrophin) levels declined to normal levels within a month, and patients were discharged without further complications in two or three hours after the procedure. Our cases show that this treatment is effective, safe, and minimally invasive for patients diagnosed with CSP.
\end{abstract}

Keywords: Ectopic Pregnancy; Cesarean Section Scar; Local MTX Treatment

\section{INTRODUCTION}

Myometrial pregnancy developing in a previous caesarean section scar is the rarest of all ectopic pregnancies (EP) and probably one of the most dangerous because of the risk of rupture and hemorrhage [1]. The recent recognition of this problem has meant that diagnosis and management are still in their infancy, and there is no consensus regarding the best management of CSP. Methotrexate can be administered systemically or locally, or in both ways, with the aid of ultrasound. If treatment with MTX fails, open or endoscopic surgery is the treatment of choice. Local MTX injection is a surgical

\footnotetext{
"Corresponding author.
}

procedure that can cause abdominal bleeding. Medical management using systemic methotrexate and local injection of embryocides has been used successfully in a number of small series; however, the time taken for serum $\beta$-hCG to return to normal levels can be prolonged [2].

This kind of treatment for CSP has not been reported before. This method is safe and effective. It offers a short operative time, short postoperative stay, and a rapid return of $\beta$-hCG to normal. We aim to share our experience [3] with a safe and short treatment modality for this increasingly common problem.

\section{CASE REPORTS}

\subsection{Case 1}

A 30-year-old woman (Gravida 2, Para 1), with one low segmental transverse cesarean section (CS) 4 years ago, presented with lower abdominal pain and vaginal bleeding after 7 weeks of her last menstruel period (LMP). Her serum $\beta$-hCG level was $15000 \mathrm{mIU} / \mathrm{mL}$. Both transvaginal and abdominal ultrasound revealed a viable singleton gestation that appeared fixed within the myometrium, anterior to the cervix and adjacent to the bladder (Figure 1).

No intrauterine pregnancy was identified. Patient was diagnosed as cesarean scar ectopic pregnancy (CSP).

Feticide was performed via intra-amniotic fluid with methotrexate (MTX) $(25 \mathrm{mg})$ injection by using a transvaginal oocyte pick-up (OPU) needle. Her $\beta$-hCG level was monitored until returned to normal levels $(\leq 5$ $\mathrm{mIU} / \mathrm{ml}$ ) on day 30 . A transvaginal ultrasound was performed four weeks after the procedure. A follow-up ultrasound showed resolution of the gestational sac. The process took about 5 minutes with no blood loss.

\subsection{Case 2}

A 33-year-old womangravida 3, Para 2 (both births by cesarean section), 6 weeks 6 days pregnant according to her last menstrual period, presented with vaginal bleeding and lower abdominal pain. USG examination revealed a gestational sac localized in the previous cesarean 


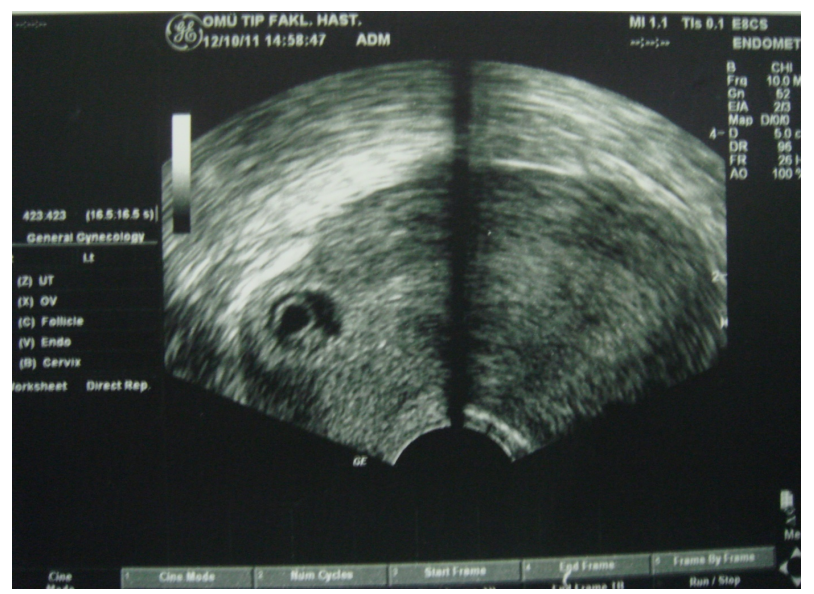

Figure 1. Case 1. A gestational sac with a positive fetal heartbeat located in the thin lower-anterior wall of the uterus behind a previous cesarean section scar as demonstrated via transvaginal sonography.

section scar. She was pregnant 7 weeks 3 days acording to Crown-rump length measurement and the fetus had heart beat. Level of $\beta$-hCG was $12,000 \mathrm{mIU} / \mathrm{mL}$.

Patient was prepared in lithotomy position and $25 \mathrm{mg}$ ofmethotrexate was administeredinto the gestational sac by an OPU needle under transvaginal USG guidance.

She was discharged 3 hours after the procedure. $\beta$ hCG level monitoring was performed weekly. $\beta$-hCG levelsreturned to normal after four weeks.

\section{DISCUSSION}

Batioğlu et al. in 1997 first described succesful treatment of cornual pregnancy with methotrexate by using transvajinal ultrasonography [3].

Cesarean scar ectopic pregnancy may be an incidental finding on routine early ultrasound in an asymptomatic woman, but it may present with acute-onset lower abdominal cramping, pain, or vaginal bleeding. The most appropriate management of cesarean scar ectopic pregnancy is not yet clear, although several treatment modalities have been proposed. Many of these modalities include expectant management, systemic and local administration of MTX, KCl, selective uterin artery embolization, hysteroscopy, D/C and even fine-needle aspiration [4].

Hysteroscopy is a minimally invasive operative technique that offers direct visualization, low morbidity, and high primary success rates to date, although numbers are small and further experience would be helpful to determine the safest and most appropriate technique. It has the distinct advantage over systemic and local injection techniques in that it affords the patient shorter follow-up time and a more rapid return to fertility with postoperative results indicating a variety of pathological findings with a variable plane of dissection and sporadic and unpredictable bleeding.

The short time interval of return to normal $\beta$-hCG levels indicates that complete removal of all gestational material is likely even when visualization is not optimal [3].

Rotas et al. reported a wedge resection of the CSP scar and repair of the defect via laparoscopy or laparotomy as a safe technique [5].

Our treatments succeeded in terminating the CSP and retaining the patient's uterus, which may assure the patient's future fertility. In our cases, the patients were not interested in conceiving in the future. After counseling, they accepted the treatment modality.

Afterwards an intra uterin device (IUD) was administered to all patients to prevent further complications like a reccurrent cesarean scar pregnancy and a possible uterine rupture in the event of pregnancy .

\section{CONCLUSIONS}

This treatment modality has the advantage of a rapid return to normal $\beta$-hCG and gives us the chance of elective laparoscopic correction of uterine scar for further fertility.

Our results show that the transvaginal treatment of ectopic pregnancy is an effective, safe, and minimally invasive procedure for patients diagnosed with CSP. This minimally invasive nonsurgical approach can preserve fertility without any major risk to the mother. In view of the increasing rate of caesarean deliveries, medical personnel should be aware of the possibility of this type of ectopic pregnancy and act accordingly.

\section{REFERENCES}

[1] Pierre-Arnaud, G., Salim, B. and Jacques, D. (1997) An ectopic pregnancy developing in a previous caesarian section scar. Fertility and Sterility, 65, 398-400.

[2] Rebecca, D. and Jason, A. (2010) Hysteroscopic management of cesarean scar ectopic Pregnancy. Fertility and Sterility, 93, 1735-1740.

doi:10.1016/j.fertnstert.2008.12.099

[3] Batığlu, S., Haberal, A., Yeşilyurt, H. and Ekici, E. (1997) Successful treatment of cornual pregnancy by local injection of methotrexate underlaparoscopic and transvaginal ultrasonographicguidance. Gynecologic and Obstetric Investigation, 44, 64-66. doi:10.1159/000291413

[4] Homayoun, S., Thomas, R., Beth, W. R., Katherine, H. C., Christina, M. D., Marsha, K. G., Pinar, H. K. and Errol, R. N. (2010) Cesarean scar ectopic pregnancy: Case series and review of the literature. American Journal of Perinatology, 27, 111-120. doi:10.1055/s-0029-1224874

[5] Rotas, M., Haberman, S. and Levgur, M. (2006) Cesarean scar ectopic pregnancies etiology, diagnosis and management. Obstetrics \& Gynecology, 107, 1373-1381. doi:10.1097/01.AOG.0000218690.24494.ce 